\title{
The needle in the haystack
}

Are all cancer cells equal or are some uniquely responsible for initiating and sustaining the growth of a tumour? Although the idea of a cancer stem cell (CSC) or tumour-initiating cell was well entrenched by the 1960s, it was not until the mid-1990s that these cells were identified and characterized.

Most of our understanding of CSCs has come from studying haematopoietic malignancies. Jacob Furth and Morton Kahn were the first to allude to CSC principles in 1937. Using cell lines, they provided the first quantitative assay for the assessment of the frequency of the malignant cell maintaining the haematopoietic tumour, at a time when the origin of leukaemia as being viral or cellular was in dispute. They showed that a single leukaemic cell was able to transmit the systemic disease when transplanted into a mouse. This was followed by the development of quantitative methods in the 1960 s and 1970 s to measure the clonogenic potential of the cell type able to sustain tumour growth in vivo. Robert Bruce and Hugo Van der Gaag used the spleen colony-forming assay (CFU-S) - a tool first developed by James Till and Ernest McCulloch, and now widely used in stem-cell biology - to show that only a small subset of primary cancer tissue was able to proliferate in vivo. Collectively, these studies underscored the functional heterogeneity in tumours - not every cell is able to proliferate to form a colony in vitro or to give rise to a tumour when transplanted in vivo - and introduced the concept of CSCs.

However, it was not until the identification and prospective purification of CSCs by John Dick and colleagues in 1994 that concrete proof was provided for a hierarchical (or stem cell) model of cancer. Using limiting dilution analysis together with disease-initiation models, these investigators showed that when isolated from acute myeloid leukaemia (AML) patients, only a small fraction of the tumour cells with a characteristic marker signature were able to establish leukaemia in recipient mice. This provided a reproducible way of enriching cells with tumour-initiating activity and ruled out the stochastic model, which predicted that such an activity would be present in every cell fraction. AML-initiating cells were not only able to differentiate and proliferate, but also had the capacity to self-renew in vivo - a key attribute of stem cells.

Recently, studies in solid tumours have revealed that the CSC concept extends beyond haematopoietic malignancies. Michael Clarke and colleagues, and Peter Dirks and co-workers showed that human breast and brain tumours are not homogeneous, but rather contain a small subset of cells that can be prospectively isolated and are able to initiate phenotypically heterogeneous cancers in vivo.

The identification of solid tumour stem cells provided researchers with a firm basis on which to re-evaluate cancer therapies to target and eliminate not only the bulk population of tumour cells, but also the rare but potent selfrenewing cells that initiate and sustain cancers. Efforts are now underway to unravel the mechanisms that regulate CSC function, and

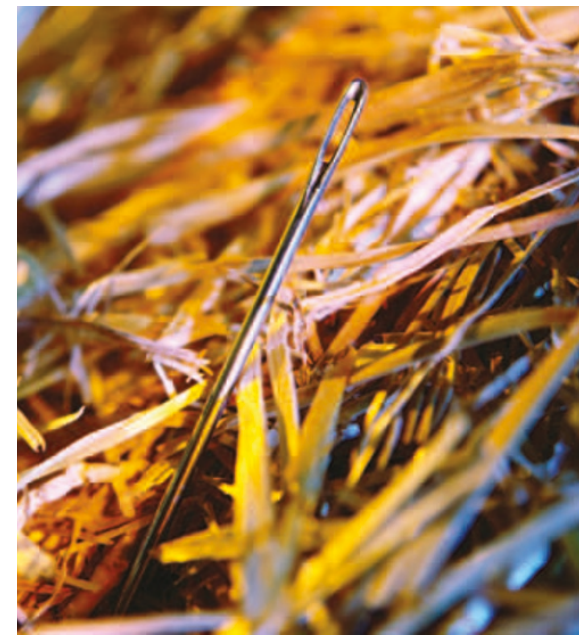

to determine whether such cells arise through mutations accrued in normal tissue stem cells or whether stem-cell properties are acquired in more differentiated progenitor cells.

Myrto Raftopoulou, Associate Editor, Nature Cell Biology

References and links

ORIGINAL RESEARCH PAPERS Furth, J. \& Kahn, M. C. The transmission of leukaemia of mice with a single cell. Am J. Cancer 31, 276-282 (1937) | Bruce, W. R. \& Van der Gaag, H. A quantitative assay for the number of murine lymphoma cells capable of proliferation in vivo. Nature 199, 79-80 (1963) | Becker, A. J., McCulloch, E. A. \& Till, J. E. Cytological demonstration of the clonal nature of spleen colonies derived from transplanted mouse marrow cells. Nature 197, 452-454 (1963) | Buick, R. N., Till, J. E. \& McCulloch, E. A. Colony assay for proliferative blast cells circulating in myeloblastic leukaemia. Lancet 1, 862-863 (1977) | Lapidot, T. et al. A cell initiating human acute myeloid leukaemia after transplantation into SCID mice. Nature 367, 645-648 (1994) | Bonnet, D. \& Dick, J. E. Human acute myeloid leukaemia is organised as a hierarchy that originates from a primitive hematopoietic cell. Nature Med. $\mathbf{3}$, 730-737 (1997) | Al-Hajj, M. et al. Prospective identification of tumorigenic breast cancer cells. Proc. Natl Acad. Sci. USA 100, 3983-3988 (2003) | Singh, S. K. et al. Identification of human brain tumour initiating cells. Nature 432, 396-401 (2004) FURTHER READING Wang, C. Y. \& Dick, J. E. Cancer stem cells: lessons from leukaemia. Trends Cell Biol. 15, 494-501 (2005)

URL

leukaemia

http://www.cancer.gov/cancertopics/types/leukemia 